\title{
Um Método para Seleção de Padrões para Melhoria de Processos de Negócio
}

\author{
Alexandre Souza ${ }^{1}$, Leonardo Guerreiro Azevedo ${ }^{1,2}$, Flavia Santoro ${ }^{1,2}$ \\ ${ }^{1}$ Departamento de Informática Aplicada - \\ Universidade Federal do Estado do Rio de Janeiro (UNIRIO) \\ Caixa Postal 22.290-240 - Rio de Janeiro - RJ - Brasil \\ ${ }^{2}$ Núcleo de Pesquisa e Prática em Tecnologia - NP2Tec \\ \{alexandre.souza, Azevedo, Flavia.santoro\}@uniriotec.br
}

\begin{abstract}
Business Process Management is increasingly present in organizations, and process improvement is one of its focuses. Patterns for process redesign have been the focus of some studies, which seek to provide solutions to recurring problems of process improvement. However, the choice of these patterns is not an easy task, especially when performed in scenarios with large models and complex processes, which makes manual analysis a very costly task. This paper aims to propose a method to assist in the analysis of these patterns by automating the choice of patterns to be applied using information present in the process models.
\end{abstract}

Resumo. Gestão de Processos de Negócio está cada vez mais presente nas organizações, e a melhoria de processos é um de seus focos. Padrões para redesenho de processos tem sido o foco de alguns trabalhos, os quais buscam apresentar soluções para problemas recorrentes de melhoria de processos. Contudo, a escolha destes padrões não é uma tarefa simples, principalmente quando executada em cenários onde se tem modelos de processos grandes e complexos, o que torna a análise manual uma tarefa muito custosa. Este trabalho tem como objetivo propor um método para auxiliar na análise destes padrões através da automatização da escolha dos padrões a serem aplicados utilizando informações presentes nos modelos de processos.

\section{Introdução}

Processo de negócio consiste em atividades coordenadas a fim de atingir um determinado objetivo. Portanto, é através da execução de processos de negócio que as organizações realizam seus propósitos Weske (2007) e Thom et al. (2007). Hammer (1990) e Davenport e Short (1990) foram os primeiros autores a discutirem uma forma sistemática de melhoria de processos.

Reijers (2005) afirma que, durante anos, boas práticas foram coletadas para melhoria de processos. Uma boa prática pode ser expressa como um padrão, que descreve uma forma de tratar um problema particular, que pode ser aplicado a situações similares. Reijers (2005) então propõe que, assumindo certos objetivos, relacionados a perspectivas de tempo, qualidade, custo e flexibilidade, um analista pode utilizar a lista de padrões, para avaliar e melhorar um processo existente. De acordo com Forster 
(2009), o uso destes padrões pode ajudar também a racionalizar e formalizar métodos para melhoria de processos.

Pourshahid (2010) aponta que a escolha destes padrões é muito difícil, e que as notações de modelos de processos não fornecem meios para se observar ou simular o impacto destes padrões nos processos, seus indicadores e objetivos. Dessa forma, o suporte para selecionar um padrão apropriado para uma situação atual do negócio é um desafio. Em um cenário onde existem processos de negócio grandes e complexos, a seleção desses padrões se torna ainda mais difícil devido ao número de elementos afetados e a complexidade embutida nos mesmos.

Por outro lado, os modelos de processos contêm muitas informações (entradas e saídas, executores, sistemas, dependências entre as atividades, eventos, etc) que podem ser utilizadas para auxiliar a seleção dos padrões. Assim, o objetivo deste trabalho é apresentar um método para seleção automática dos melhores padrões a serem utilizados para melhoria de processos a partir da leitura de modelos de processos de negócio.

O artigo está organizado da seguinte forma: a Seção 2 apresenta os padrões de redesenho de processo; a Seção 3 descreve um método existente para tomada automática de decisões a partir de modelos de processos de negócio, que será tomado como base nesta proposta; na Seção 4, é descrita a proposta de solução; a Seção 5 discute um cenário de aplicação e a Seção 6 conclui o artigo.

\section{Padrões de redesenho de processos}

De acordo com Reijers (2005), um padrão é uma boa prática que descreve uma forma de tratar um problema particular, e que pode ser aplicado em situações similares. Reijers (2005) fornece um survey contendo diversos padrões de redesenho de processos, apresentando suas características, o impacto que cada um causa no processo e algumas situações comuns que favorecem sua aplicação. Os padrões são baseados em perspectivas de tempo, custo, qualidade, e flexibilidade.

A perspectiva de tempo diz respeito ao tempo gasto para se levar um caso (instância do processo) do início ao fim. Existem muitas formas de abordar esta perspectiva, como por exemplo, tentar diminuir o tempo médio gasto no processo ou o tempo máximo de cada caso, ou até mesmo a variação do tempo entre os casos.

A perspectiva de custo está relacionada à questão financeira. É comum que o foco de melhoria seja em reduzir custos operacionais, particularmente em recursos humanos. Automação é comumente vista como uma alternativa, contudo outros custos estão envolvidos, como por exemplo, o custo de desenvolvimento e manutenção da aplicação.

A perspectiva de qualidade geralmente está associada à qualidade interna e externa. A qualidade externa é a satisfação do cliente em relação ao produto e ao processo. A qualidade interna está ligada a condições de trabalho e controle do trabalho. Todas as perspectivas que buscam melhorar custo em geral pioram a qualidade.

A perspectiva de flexibilidade pode ser definida como a habilidade de reagir a mudanças, como, por exemplo, gerenciamento de mudanças na estrutura do processo e alocação de recursos, e mudanças na estrutura e responsabilidade dos processos para atingir necessidades do mercado e dos parceiros de negócio. 
Além destas perspectivas, os padrões são classificados por Reijers (2005) em: (i) Melhores práticas em atividades: Foca em otimizar uma única atividade em um processo de negócio; (ii) Melhores práticas em roteamento: Tenta melhorar a estrutura de roteamento do processo de negócio; (iii) Melhores práticas em alocação: Envolve a alocação particular de recursos no processo de negócio; (iv) Melhores práticas em recursos: Foca nos tipos e números de recursos; (v) Melhores práticas em terceiros: Tenta melhorar a colaboração e comunicação com o cliente e terceiros; (vi) Melhores práticas em integração: Se aplicam ao processo de negócio como um todo. Reijers (2005) apresenta 28 padrões relacionados às classificações acima, suas características e situações de uso. A seguir, é apresentado um exemplo para cada tipo de padrão.

Composição de atividades (classe "melhores práticas em atividades") tem como propósito reunir atividades. Ela combina atividades finas (atividades que consistem em poucos procedimentos) em uma única atividade, ou divide atividades grossas (atividades que consistem em muitos procedimentos) em atividades finas trabalháveis. Combinar atividades deveria resultar em redução de tempo de iniciação, que é o tempo gasto pelo recurso para se tornar familiar com um caso específico. Atividades muito grossas podem resultar em diminuição da flexibilidade e piora da qualidade, já que as atividades podem se tornar impraticáveis. Contudo, obviamente atividades menores podem resultar em um tempo maior para inicializá-las. Um exemplo de esquema de atividades e aplicação do padrão é apresentado na Figura 1.

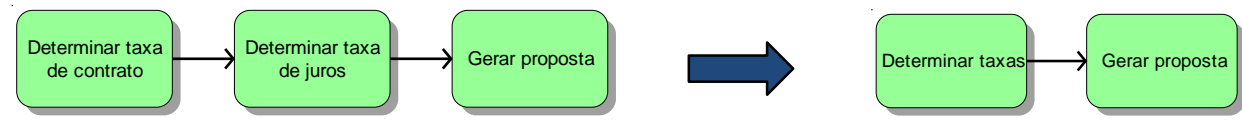

Figura 1 - Exemplo do padrão Composição de atividades

Paralelismo (classe "melhores práticas em roteamento") tem como propósito considerar se atividades podem ser executadas em paralelo. $\mathrm{O}$ efeito óbvio da aplicação desta boa prática é que o tempo de espera seria reduzido, por outro lado isto pode aumentar o custo de execução do processo. Um exemplo de esquema de atividades e aplicação do padrão é apresentado na Figura 2.

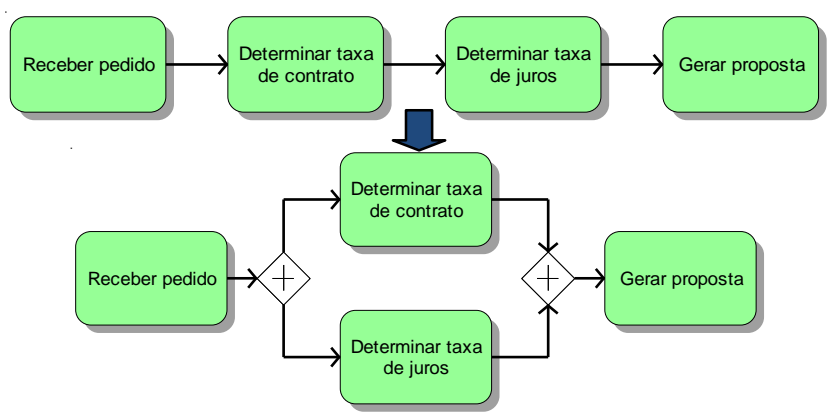

Figura 2 - Exemplo do padrão Paralelismo

Dividir responsabilidades (classe "melhores práticas em alocação") propõe evitar a atribuição de responsabilidades a pessoas de diferentes unidades funcionais a uma mesma atividade. A ideia por trás desta boa prática é que atividades, que diferentes departamentos compartilham responsabilidades sobre a mesma, costumam ser fontes de negligência e conflitos. Com esta boa prática, deveríamos ter uma melhora na qualidade de execução das atividades. Contudo, diminuir o número efetivo de recursos disponíveis 
para uma atividade pode resultar um efeito negativo no que diz respeito ao tempo. Um exemplo de esquema de atividades e aplicação do padrão é apresentado na Figura 3.
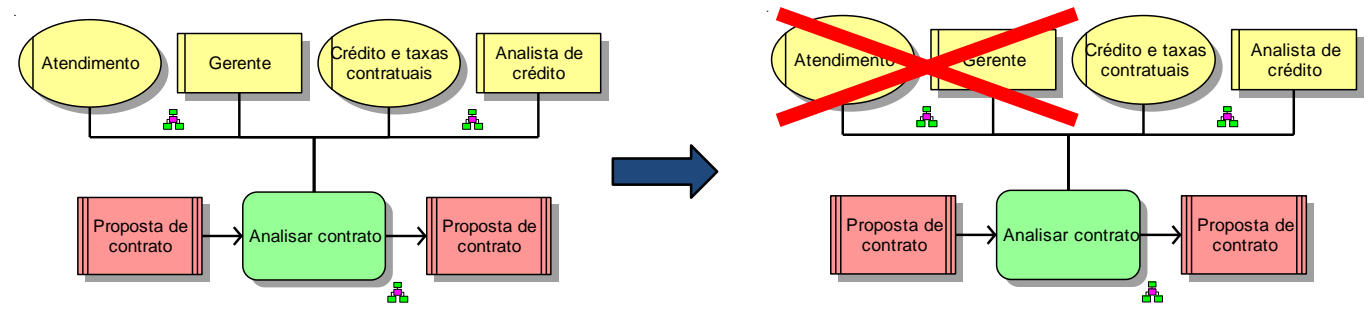

Figura 3 - Exemplo do padrão Dividir responsabilidades

Recursos extras (classe "melhores práticas em recursos") propõe considerar o aumento do número de recursos de uma determinada classe de recursos se a capacidade não estiver suficiente. $\mathrm{O}$ efeito óbvio da aplicação desta boa prática é a diminuição do tempo, e contratar novas pessoas ou comprar recursos extras aumenta o custo. Um exemplo seria uma atividade de manutenção que é executada por 1 operador de máquina que passaria a ser executada por 2 operadores de máquina.

Redução de contatos (classe "melhores práticas em terceiros") tem como objetivo reduzir o número de contatos com clientes e terceiros. A troca de informações com clientes ou terceiros sempre consome tempo. Cada contato também introduz a possibilidade de erros. Com isso, a redução de contatos deve diminuir o tempo gasto e melhorar a qualidade. A desvantagem de diminuir o número de contatos é que pode haver perda de informações essenciais, que está diretamente ligada à qualidade. Um exemplo de esquema de atividades e aplicação do padrão é apresentado na Figura 4.
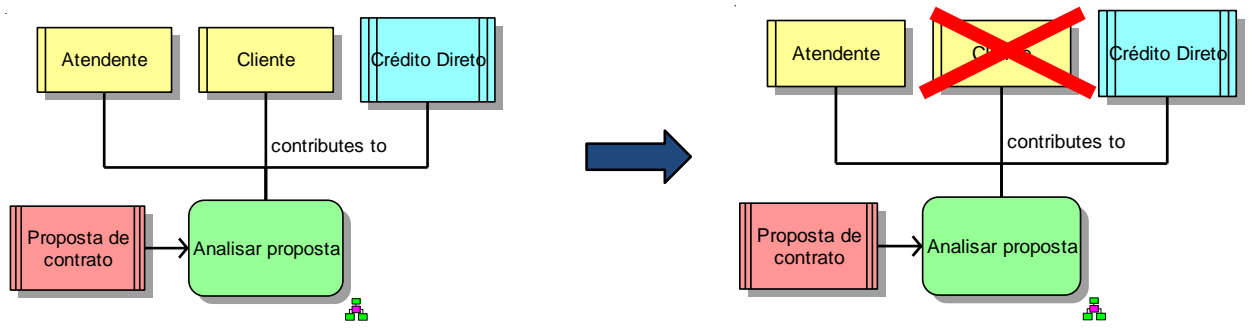

Figura 4 - Exemplo do padrão Redução de contatos

Baseado em casos de trabalho (classe "melhores práticas em integração") propõe considerar remover o processamento em lotes e as atividades periódicas do processo. Exemplos deste caso referem-se ao empilhamento de itens de trabalho em lotes para serem processados posteriormente. Evitar estes processamentos periódicos deve aumentar significantemente o tempo de cada caso, já que seria processado caso a caso em tempo real. $\mathrm{O}$ custo de manter sistemas permanentemente disponíveis para o processamento caso a caso pode aumentar em relação ao processamento periódico. Um exemplo de esquema de atividades e aplicação do padrão é apresentado na Figura 5. 


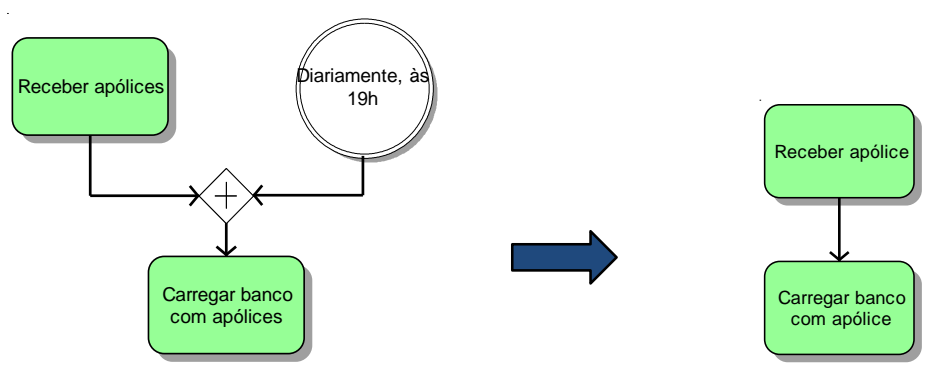

Figura 5 - Exemplo do padrão Baseado em casos de trabalho

Pourshahid (2007) apresentou uma lista contendo os impactos que cada padrão pode causar no processo em relação às quatro perspectivas (tempo, custo, qualidade $\mathrm{e}$ flexibilidade). Por exemplo: O padrão de nome "Eliminação de atividades" altera o processo de forma positiva na perspectiva tempo e custo, porém pode haver piora na qualidade, e na perspectiva flexibilidade a princípio não há alteração.

Nesta seção foram apresentados os padrões levantados por Reijers (2005). A escolha destes padrões apresentados nesta seção necessita que o analista passe por todos os padrões analisando se a sua aplicação é favorável ou não ao processo. Isto introduz a necessidade de uma maneira mais sistemática e automatizada para auxiliar o analista na decisão de uso destes padrões. Na próxima seção, é descrito um método para tomada de decisão em processos de negócio que foi utilizado como base para esta proposta.

\section{Suporte à decisão em processos de negócios}

Van Putten et al. (2011) propuseram um método de suporte à decisão sobre questões relacionadas aos elementos de um processo através de análise automática de modelos de processos de negócio. A entrada para o método é um modelo de processo, e então são geradas, com base em uma série de heurísticas e regras de negócio, recomendações para a solução das questões levantadas sobre os elementos do processo representado.

O método consiste nas cinco etapas apresentadas na Figura 6. Na primeira etapa, o método lê o modelo de processo. Na segunda etapa, são aplicadas determinadas heurísticas para levantar características do processo. Na terceira etapa, são aplicadas as regras definidas para recomendação. $\mathrm{Na}$ quarta etapa, resoluções de conflitos são aplicadas, que consiste em aplicações de pesos para as definições feitas pelas regras na etapa anterior. Por fim, na quinta etapa, são geradas as recomendações finais baseadas nos resultados das etapas anteriores.

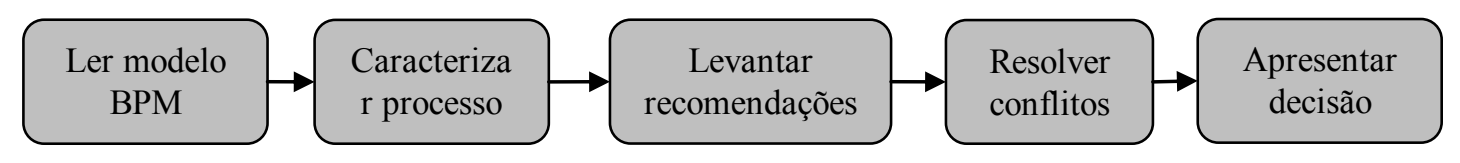

Figura 6 - Método para suporte à decisão automatizada (Van Putten et al., 2011)

Um exemplo de decisão apresentado pelos autores é o local de disponibilização de uma informação sobre o produto gerado no processo. O produto é uma garrafa de vinho e as informações podem estar na própria garrafa ou referenciada para outro local. O trabalho mostra que os processos contêm informações que auxiliam na tomada de 
decisão. No exemplo, o processo utilizado como entrada descreve como o produto é utilizado e qual a intensidade de uso dos dados. A partir do modelo do processo, heurísticas e critérios de resolução de conflitos, gerados especificamente para a tomada de decisão sobre o local de armazenamento, apontam recomendações para onde a informação deve ser disponibilizada.

As etapas executadas para o exemplos são apresentadas na Figura 7. Na primeira etapa, o modelo de processo é lido. Na segunda etapa, são aplicadas heurísticas que buscam no modelo características que determinam, por exemplo, a freqüência de acesso ao dado do produto e disponibilidade de internet no momento. Na terceira etapa, são aplicadas as regras definidas para recomendação. Neste caso, as regras são: (i) se as informações do produto são muito acessadas, então é recomendado armazená-las na própria garrafa; caso contrário, as informações devem ser referenciadas; (ii) se houver disponibilidade de internet no momento, então a informação deve ser armazenada em um site, caso contrário (não houver disponibilidade de internet), então as informações devem ser armazenada localmente na garrafa. Na quarta etapa de resoluções de conflitos, foi definido que a freqüência de acesso ao dado tem peso maior que disponibilidade de internet, logo se tiver internet disponível no momento e o dado for muito acessado, é sugerido que a informação seja armazenada localmente, pois o acesso é o que predomina. Por outro lado, se o dado for pouco acessado, mas houver disponibilidade de internet, é sugerido que a informação seja referenciada. Por fim, na quinta etapa, as recomendações finais são apresentadas para o analista decidir.

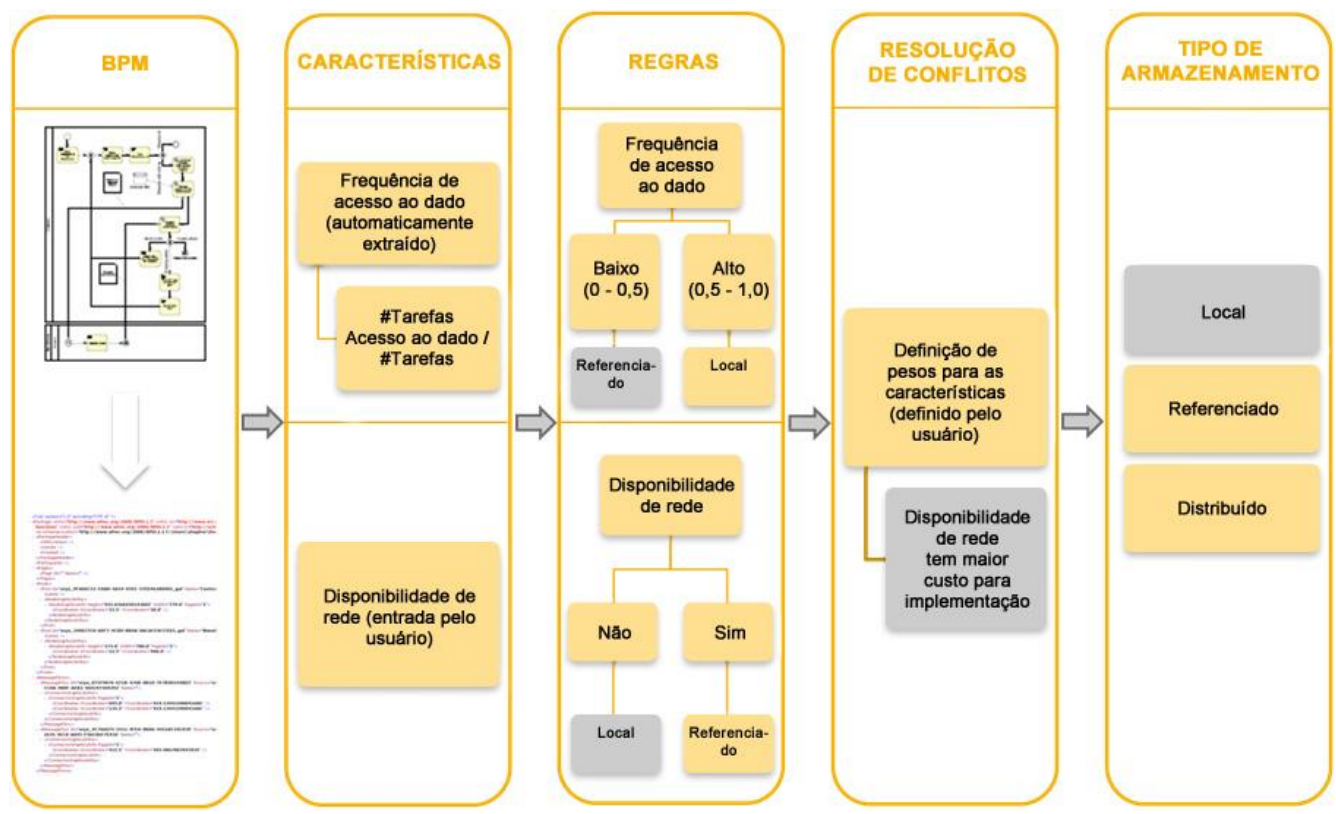

Figura 7 - Exemplo de aplicação do méto para suporte à decisão automatizada (adaptado de Van Putten et al., 2011)

No nosso trabalho, o método proposto por Van Putten et al. (2011) é adaptado para identificar, a partir de modelos de processos de negócio, quais padrões de melhoria de processos são favoráveis ou desfavoráveis para serem aplicados, e assim auxiliar o analista na tarefa de decidir como melhorar o processo em questão. 


\section{Proposta: Um Método para Melhoria de Processos baseado em Padrões de Redesenho}

O método proposto é uma adaptação do trabalho de Van Putten et al. (2011) para auxiliar o analista a selecionar os padrões apresentados por Reijers (2005) para melhoria de processos. De acordo com a nossa premissa, os modelos de processos, entrada para o método, contêm informações que podem auxiliar na seleção destes padrões. O objetivo geral do método é aplicar heurísticas elaboradas para identificar os problemas típicos descritos nos padrões, e assim, sugerir se a aplicação de determinado padrão é favorável ou não ao processo em questão.

O método é composto pelas cinco etapas apresentadas na Figura 8. Na Etapa 1 (BPM - Business Process Model), o processo de negócio é lido, em seguida na Etapa 2 (Heurísticas), são aplicadas as heurísticas elaboradas neste contexto, para identificação de problemas presentes no modelo. A Etapa 3 (Recomendações) gera recomendações de padrões a serem aplicados de acordo com os problemas presentes no modelo. Na Etapa 4 (Resolução de Conflitos), o analista define um peso para cada recomendação, para tratar possíveis conflitos entre as recomendações geradas. Por fim, na Etapa 5 (Recomendações Finais), os resultados anteriores são considerados para apresentação de um ranking das recomendações finais de padrões a serem aplicados no processo.

As principais contribuições deste trabalho estão na definição e sistematização do conjunto de heurísticas para analisar um modelo de processos de negócio, observando alguns de seus elementos e informações relacionadas, a fim de identificar os problemas relatados nos padrões de redesenho e sugerir quais são os padrões mais apropriados de serem aplicados para melhorar o processo. Os padrões descritos por Reijers (2005) são descritos em linguagem informal (linguagem natural), desta forma, é necessário formalizar estes enunciados e relacionar os elementos que os compõe com os elementos ou objetos do modelo de processo em questão. A seguir, alguns exemplos de heurísticas são apresentados a fim de ilustrar nossa proposta.

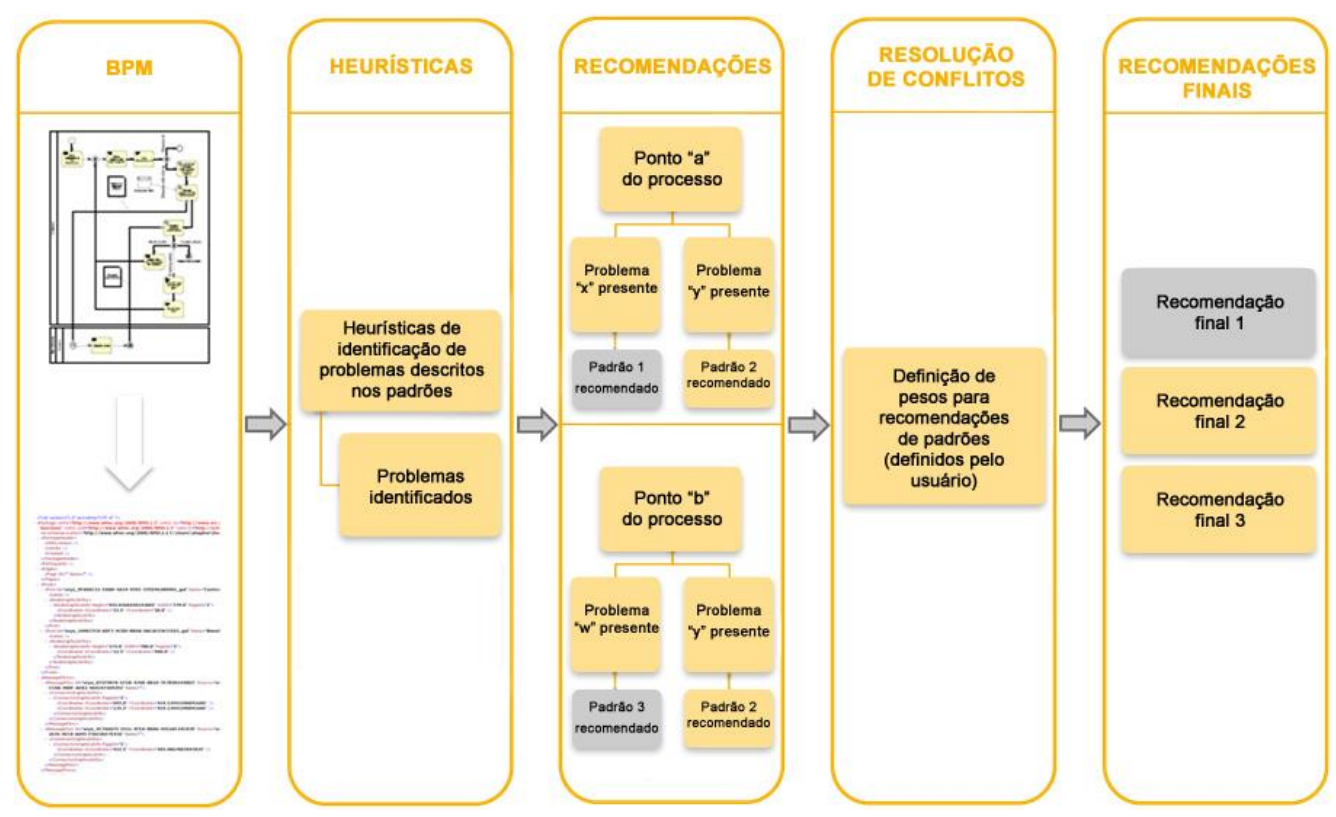

Figura 8 - Método para melhoria de processos baseado em padrões de redesenho 
A heurística H1 Atividade Sequencial tem como propósito buscar atividades seqüenciais que podem ser executadas em paralelo, de acordo com o problema relatado no padrão Paralelismo. A heurística deve ser aplicada para toda sequência de atividades no modelo (sequência de atividades tipicamente são modeladas em modelos de processos como a ligação entre duas atividades sem controle de fluxo condicional (Van der Aalst et al., 2003)).

H1 Atividade sequencial: Dadas atividades sequenciais no modelo, caso não haja algum insumo utilizado como entrada numa das atividades sequenciais que tenha sido produzido como saída na atividade anterior a esta, a aplicação do padrão "paralelismo" neste ponto do modelo é favorável

A heurística H2 Responsabilidade Compartilhada tem como objetivos identificar a atribuição de responsabilidades a pessoas de diferentes unidades funcionais a uma mesma atividade. A heurística deve ser aplicada para todas as atividades do modelo.

$\mathrm{H} 2$ Responsabilidade compartilhada: Se uma mesma atividade é realizada ao mesmo tempo por mais de uma unidade organizacional, então a aplicação do padrão "dividir responsabilidades" é favorável.

A heurística H3 Atividade Composta tem como objetivo buscar atividades grandes ou complexas. Esta heurística deve ser aplicada para todas as atividades do modelo.

H3 Atividade composta: Caso uma atividade possua muitas saidas (a quantidade pode ser definida pelo analista) - isto significa que muitos produtos são gerados nesta atividade -, então a aplicação do padrão "composição de atividades" é favorável para realizar a divisão desta atividade em atividades menores.

A heurística H4 Atividade Simples tem como objetivo buscar atividades pequenas ou que não agregam valor ao processo. Esta heurística deve ser aplicada para todas as atividades do modelo.

H4 Atividade simples: Caso uma atividade não possua nenhuma saída, isso significa que a atividade é simples de mais, então a aplicação do padrão "composição de atividades" é favorável para realizar a composição desta atividade com a atividade exatamente anterior.

A partir das recomendações, o analista foca seu trabalho na análise das mesmas, ao invés de passar por cada um dos padrões analisando todo o modelo. Neste momento, ele poderia decidir com base em sua experiência, conhecimento e habilidades quais recomendações dadas pelo método são aplicáveis no seu cenário em questão.

\section{Cenário de aplicação do método proposto}

Utilizamos o processo "Analisar pedido de crédito" apresentado por Souza et al. (2010) e ilustrado na Figura 9 para avaliação do método proposto neste trabalho. O processo inclui 18 atividades conduzidas por 2 departamentos em uma organização na área financeira. Este processo analisa propostas de crédito, as quais podem ser aprovadas ou negadas. O processo foi modelado utilizando a notação EPC (Cheer, 2000), sendo os fluxos de atividades modelos como eEPC (extended Event Process Chain) e cada atividade detalhada em diagramas chamados de FAD (Function Allocation Diagram). Por limitação de espaço, apenas o fluxo eEPC é apresentado (Figura 9), não sendo 
apresentados neste trabalho os FADs das atividades. As técnicas para modelagem de processos propostas por Sharp e McDemortt (2001) foram utilizadas na modelagem do processo. As marcações pontilhadas não fazem parte da linguagem, foram incluídas na Figura 9 a fim de ressaltar aspectos que serão tratados na aplicação do método.

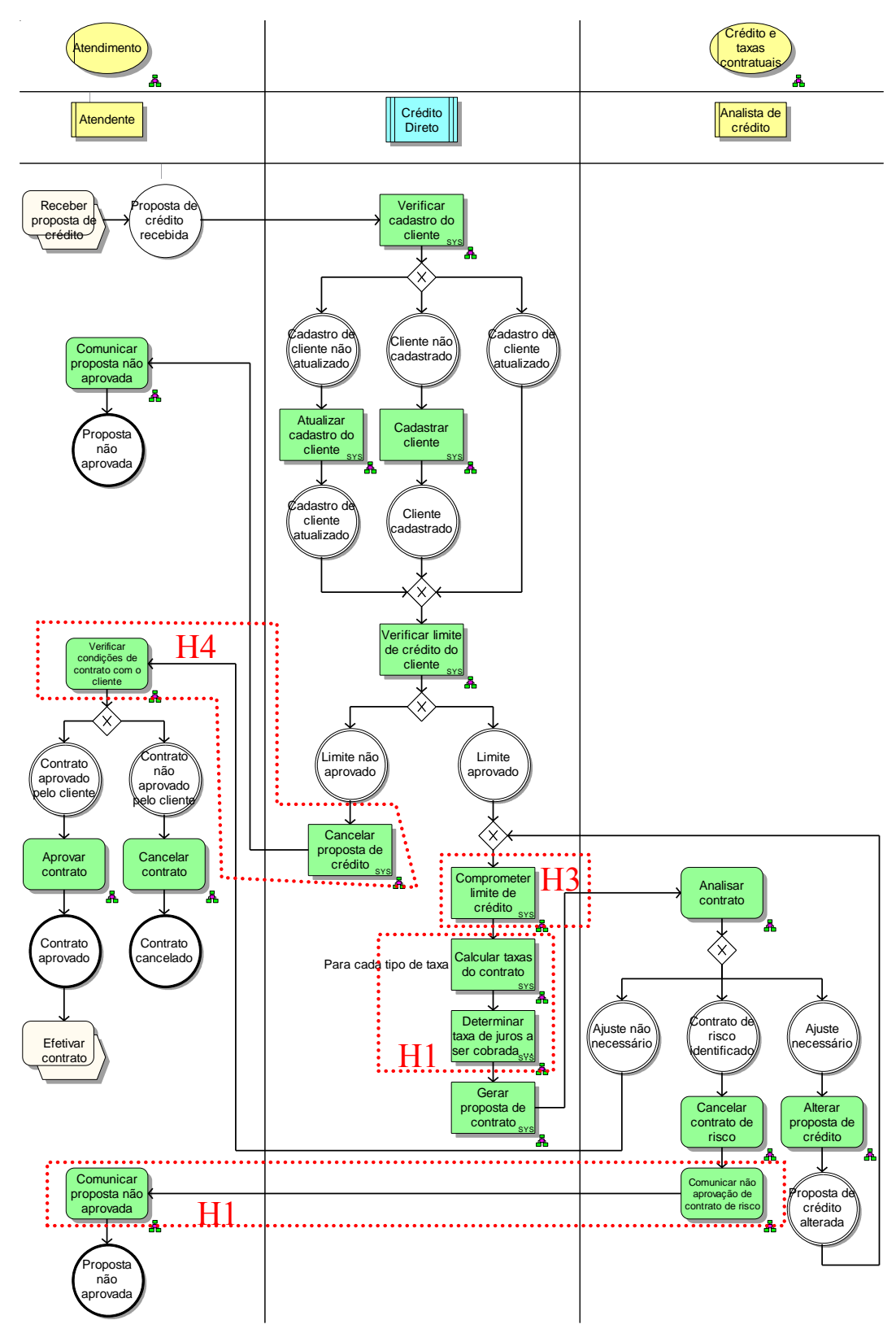

Figura 9 - Processo "Analisar pedido de crédito"

O processo inicia quando uma proposta de crédito é recebida, então as informações do cliente são analisadas e o sistema verifica se o limite de crédito do cliente é compatível com a proposta submetida. Se o limite for compatível, então o sistema calcula os impostos e taxas a serem geradas para a proposta de contrato. Esta proposta de contrato é enviada para um analista que verifica se há necessidade de ajustes e define o nível de risco da proposta. Se o contrato for aceito, o atendente entra em contato com 
o cliente. Se o contrato for aprovado pelo cliente, o processo de efetivação do contrato é iniciado. Caso contrário, o contrato é cancelado.

Aplicando o método proposto, primeiramente o modelo do processo foi lido e em seguida as heurísticas definidas na seção anterior foram aplicadas. Os pontos do processo nos quais as aplicações de padrões foram recomendadas foram marcados na Figura 9, em retângulos vermelhos pontilhados.

Heurística H1 Atividade sequencial: as atividades "Calcular taxas do contrato" e "Determinar taxa de juros a ser cobrada do cliente" seriam recomendadas a serem executadas em paralelo por não existirem insumos na segunda atividade que tenham sido produzidos na primeira. A mesma situação ocorre para as atividades "Comunicar não aprovação de contrato de risco" e "Comunicar proposta não aprovada".

- Heurística H2 Responsabilidade compartilhada: não foi identificado nenhum ponto do processo para aplicação do padrão, pois não existe nenhuma atividade com mais de uma unidade organizacional responsável.

- Heurística H3 Atividade composta, foi recomendado que a atividade "Comprometer limite de crédito" fosse dividida em atividades mais finas por possuir muitas saídas.

- Heurística H4 Atividade simples: apenas a atividade "Verificar condições de contrato com o cliente" seria recomendada para ser composta com a atividade anterior por não possuir nenhuma saída.

Neste cenário, não houve conflito entre as recomendações geradas, e com isso não foi necessário definir pesos para as recomendações conflitantes. Com isso, as recomendações geradas foram consideradas como sendo as recomendações finais.

Uma vez aplicado o método e de posse destas recomendações, cabe ao analista verificá-las e definir quais serão aplicadas e quais serão descartadas. $\mathrm{O}$ trabalho anterior de verificar todos os pontos do processo para todos os padrões existentes foi automatizado através da aplicação do método.

Com base nas recomendações fornecidas pelo método, foi realizado o trabalho do analista de analisar as recomendações fornecidas pelo método e o modelo resultante é apresentado na Figura 10. Os 2 pontos recomendados para o padrão "Paralelismo" (Heurística H1) foram implementados no processo, por serem atividades que realmente não dependem uma da outra, e, portanto, poderiam ser executadas de forma paralela. A atividade recomendada para divisão em atividades mais finas (Heurística H3) foi dividida em 2 atividades, onde uma ficou de responsabilidade por comprometer o limite de crédito do cliente no sistema, e outra por atualizar essa informação na proposta de crédito. A atividade recomendada para composição com alguma outra atividade não foi acatada, pois embora não possua nenhuma saída na atividade, a mesma está representando uma verificação necessária para continuação do processo, não podendo ser incorporada em outra atividade. 


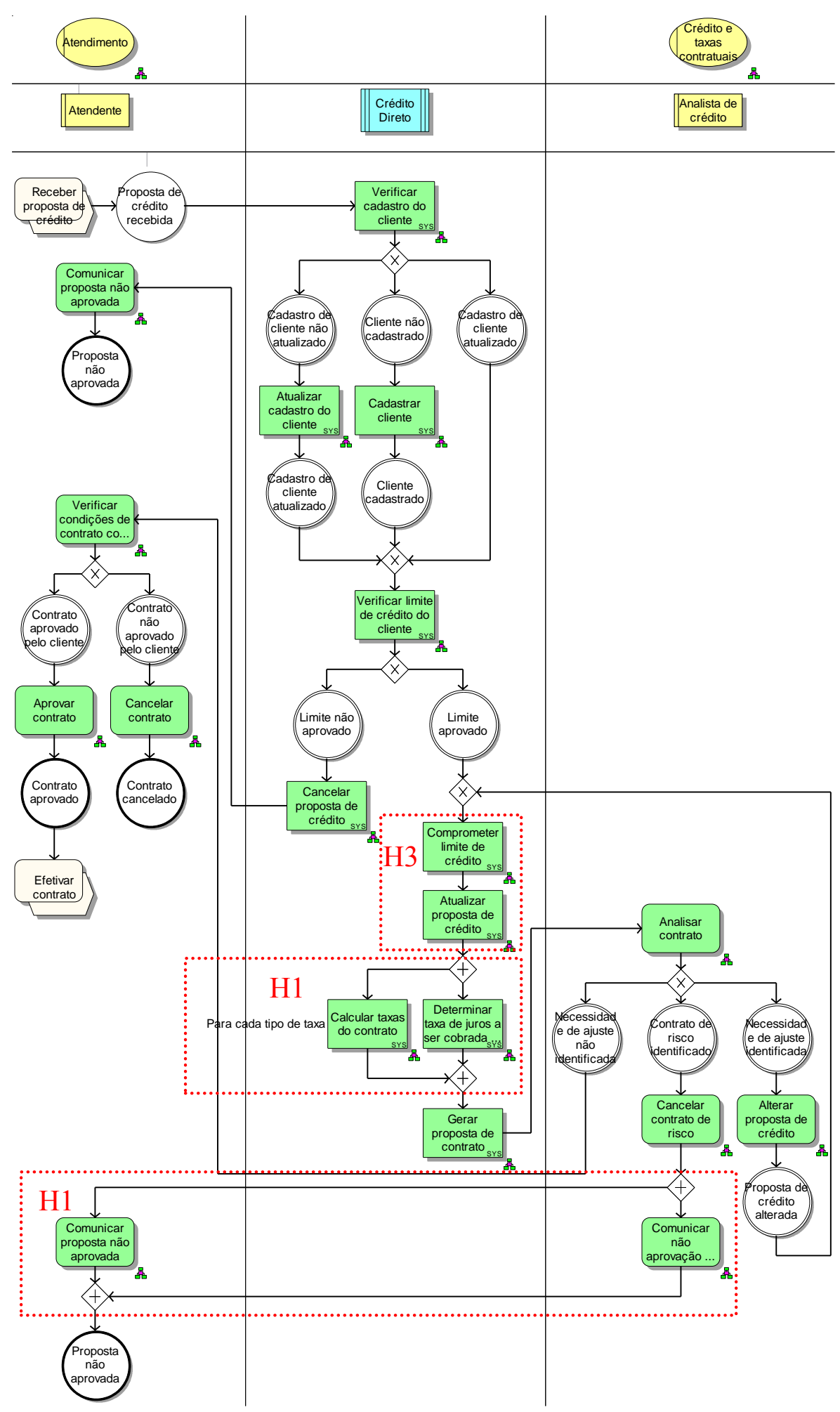

Figura 10 - Processo resultante da aplicação dos padrões

\section{Conclusões e Trabalhos Futuros}

Há cerca de mais de duas décadas se tem olhado para processos de negócio pensando em melhoria. Reijers (2005) reuniu uma série de padrões utilizados para melhoria de 
processos. Este artigo apresentou uma proposta de um método para auxiliar o analista a na selecão destes padrões. Este método visa facilitar o trabalho do analista evitando o trabalho de passar por todos os padrões analisando o modelo do processo, que num cenário contendo processos grandes e complexos pode-se tornar algo impraticável.

Como contribuição, a proposta fornece a elaboração das heurísticas para verificação dos problemas presentes no modelo de processo de negócio e seleção dos padrões favoráveis à aplicação, a resolução de conflitos entre as heurísticas, e a aplicação do método como exemplo, utilizando um processo de negócio do setor financeiro.

Como trabalhos futuros, pretende-se: definir algoritmos para implementação das heurísticas, definir melhorias das heurísticas; elaborar novas heurísticas; aplicar o método em um modelo de processo real, maior e mais complexo; e, analisar a aplicação sucessiva do método em um modelo já melhorado com o auxílio do próprio método.

\section{Referências}

Van der Aalst, W.M.P., Ter Hofstede, A.H.M., Kiepuszewski, B., Barros, A.P. (2003) "Workflow patterns". Distributed and Parallel Databases 14, 5-51.

Davenport, T. H., Short, J. E. (1990) "The New Industrial Engineering: Information Technology and Business Process Redesign”. Sloan Management Review, 31(4):1127.

Forster, F. (2009) “The Idea behind Business Process Improvement: Toward a Business Process Improvement Pattern Framework". http://www.bptrends.com/publication

files/04-06-ART-PatternFramework-Forster.pdf (acessado em Novembro 2011)

Hammer, M. (1990) “Reengineering Work: Don’t Automate, Obliterate". Harvard Business Review,70-91.

Pourshahid, A., Mussbacher, G., Amyot, D., Weiss, M. (2009) “An Aspect-Oriented Framework for Business Process Improvement". E-Technologies: Innovation in an Open World, Vol. 26. Springer Berlin Heidelberg, p. 290.

Pourshahid, A., Amyot, D., Chen, P., Weiss, M., Forster, A.J. (2007) "Business Process Monitoring and Alignment: An Approach Based on the User Requirements Notation and Business Intelligence Tools". In WER 80-91.

Van Putten, B.-J., Romeiro, C., Azevedo, L. G. (2011) "Decision Support By Automatic Analysis of Business Process Models". ECIS 2011 Proceedings.

Reijers, H. (2005) "Process Design and Redesign". In: Process-Aware Information Systems, pp. 207-234. John Wiley \& Sons, Inc., Hoboken, New Jersey, USA.

Sharp, A., McDemortt, P. (2001) "Workflow Modeling: Tools for Process Improvement and Application Development". Artech House computing library.

Souza, A., Diirr, T., Azevedo, L. G., Santoro, F. (2010). "Identificação e Análise de Serviços a partir de Modelos de Processos de Negócio: Um estudo de caso". Relatórios Técnicos do DIA/UNIRIO (RelaTe-DIA), RT-0001/2011.

Thom, L.H, Iochpe, C., Chiao, C. (2007) "Padrões de Workflow para Reuso em Modelagem de Processo de Negócio”. In: Conferência Latino Americana em Linguagens de Padrões para Programação, Porto de Galinhas, Brasil.

Weske, M. (2007) "Business Process Management - Concepts, Languages, Architectures", Verlag; Berlin; Heidelberg: Springer, 368p. 